\title{
Information Transfer Capability and Signal Processing Performance of Modern Scanning Electron Microscopes
}

\author{
Zsolt Radi, András E. Vladár, and Michael T. Postek [1] \\ National Institute of Standards and Technology, 100 Bureau Drive, Gaithersburg, MD 20899, USA
}

The scanning electron microscope (SEM) produces images with signals generated through the interaction of the primary electrons and the sample material. The proper choice of electron beam forming and imaging parameters is essential to collect relevant information. Incorrect or insufficient information leads to loss of control over the monitored phenomena. Acquisition of too much or unnecessary data results in decreased measurement speed and higher cost of operation. There are three major subsystems defining the measurement and information processing performance in the SEM. These are the electron optical column (including the electron gun and beam scanning system), the sample stage, and the signal electronics. All these must work sufficiently well to achieve the best performance.

There are several limits to the information collecting capability of SEMs. The first is the spatial resolution, which is determined by the beam quality and the mechanism of signal generation. The second is due to limited pixel and gray-scale resolutions. These have to fulfill the criteria of proper sampling and signal handling. Usually there are also limits to transferring the information across the signal chain of the measuring instrument, which includes the electron detector, the analog amplifier and signal conditioning unit, the analog-digital converter and the digital signal processing circuits.

Information theory can reveal the influence of various basic SEM properties on the measurement quality. Information transfer capability (ITC) is the ability of the microscope to turn the observed physical phenomena into a digital image. It is determined by the number of pixels in an image and the dynamic signal-to-noise ratio (DSNR) of the video signal. The DSNR is a function of the electron probe current, dwell time and the optical transfer function of the SEM, which contains all pertinent column and gun parameters. Since the ITC and the measurement resolution of the SEM are strongly related, we developed a new, more detailed model for their assessment. There are other models in the literature, that deal with various aspects of these issues to a different extent, while this new model gives a more comprehensive approach. Figure 1a shows the gray-scale resolution (the number of analog-digital conversion bits) necessary to transfer the useful physical information with maximum fidelity as a function of the linear image size measured in pixels. It also gives a comparison of the results calculated by different theoretical models. Figure $1 \mathrm{~b}$ shows comparisons of the ITC of a real and an ideal SEM equipped with an 8-bit analog-digital converter. At a given magnification and frame time, the amount of information produced by an ideal SEM increases quadratically with the linear image size. The Reimer model [2] predicts a linear increase of useful information content, while other models like Joy's signal-to-noise ratio calculations [3] deal with only a part of the contributing factors and show saturation at sufficiently high number of pixels. The new model presented here predicts earlier saturation and an even smaller amount of useful information.

The measurement performance of SEMs strongly depends on their signal processing as well. Without proper analysis of the signal chain, SEM users are not able to fully determine the presence and the extent of possible errors. Figure 2 illustrates the shortcomings of the signal processing performance 
of a state-of-the-art SEM. Figure 2a shows the transfer characteristic and non-linearity of the analogdigital signal chain measured in high-resolution modes with different types of signal conditioning. Figure $2 \mathrm{~b}$ reveals a complex distortion pattern, which was obtained as a difference of test signal applied at the analog input circuit of the microscope and the digital output data. Figure 2c is a Fourier power spectrum of the digital output data of a two-tone measurement, which shows an intermodulation (IM) distortion of about $0.6 \%$.

Similar calculations and measurements can be done for any SEM. The ITC and measurement resolution of existing instruments can be improved with the proper choice of microscope parameters whereas the newly designed SEMs should include electron beam forming and data collection systems optimized for maximum useful information transfer.

\section{References}

[1] Contribution of the National Institute of Standards and Technology (NIST), not subject to copyright.

[2] L. Reimer, Image Formation in Low-voltage Scanning Electron Microscopy, SPIE Optical Engineering Press, Bellingham, 1993.

[3] D.C. Joy, Current State of Defect Review by Electron Beam Tools: A White Paper, International SEMATECH Technology Transfer Document \#00013877A-ENG, Austin, 2000.

[4] This research was partially supported by the International SEMATECH and the NIST OMP.
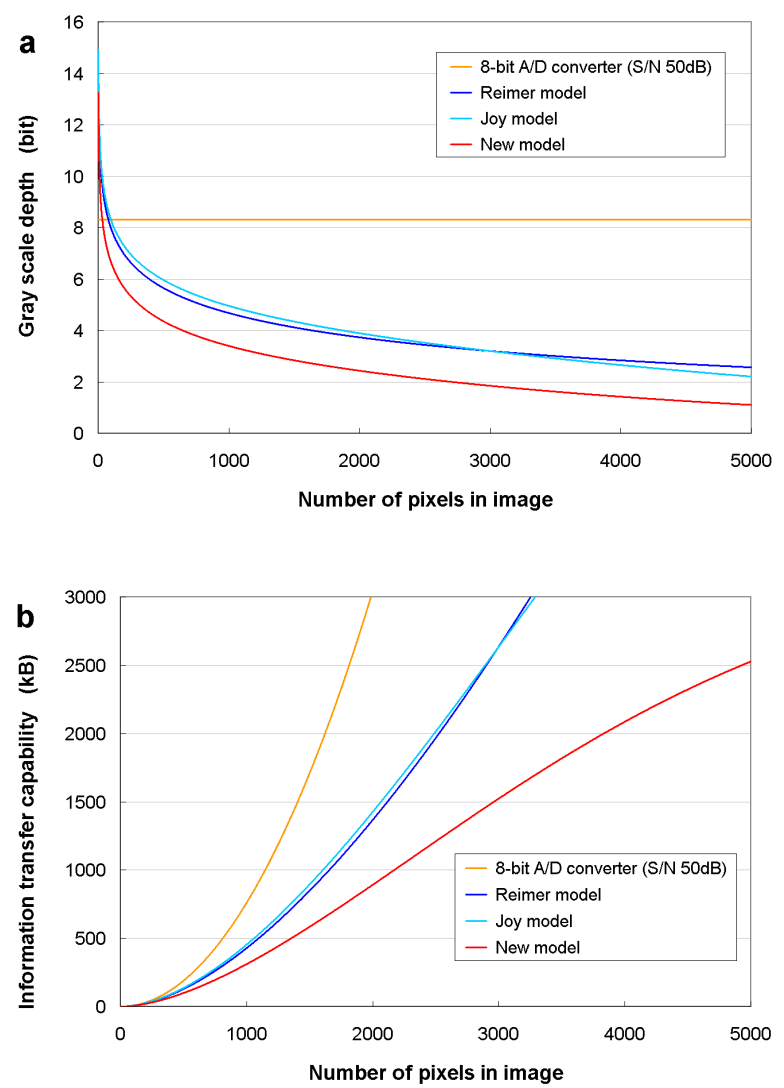

FIG. 1. Theoretical assessment of the information transfer capability of a modern SEM.
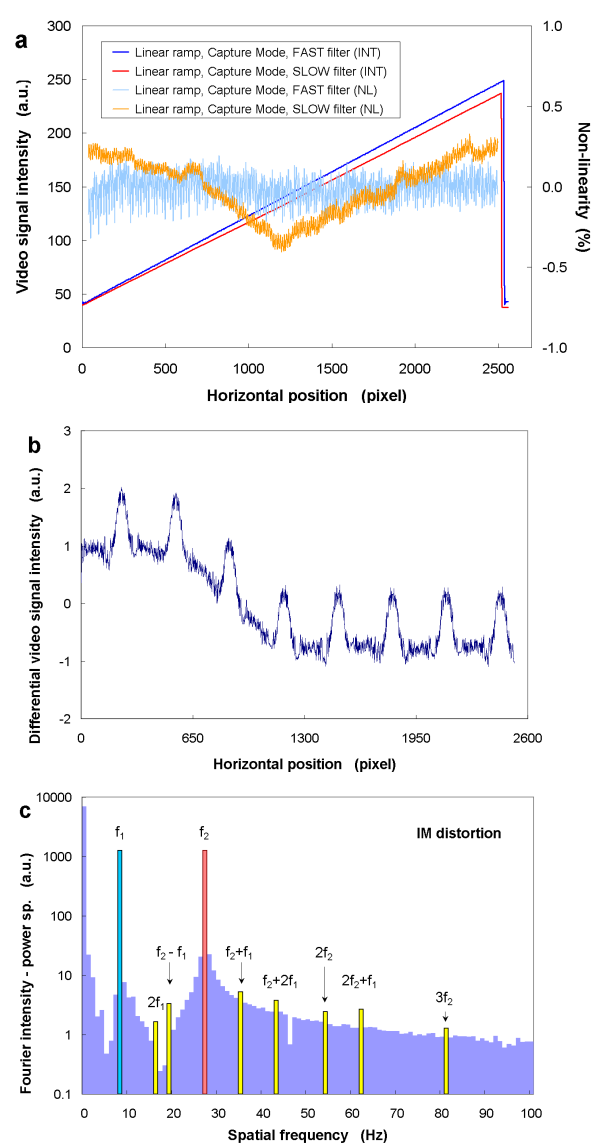

FIG. 2. Evaluation of the signal processing performance of the same instrument. 